\title{
Exact inference for the risk ratio with an imperfect diagnostic test
}

\author{
J. REICZIGEL ${ }^{1 *}$, J. SINGER ${ }^{2}$ AND ZS. LANG ${ }^{1}$ \\ ${ }^{1}$ University of Veterinary Medicine Budapest, Hungary \\ ${ }^{2}$ Accelsiors CRO \& Consultancy Services Ltd, Budapest, Hungary
}

Received 7 February 2016; Final revision 8 August 2016; Accepted 10 August 2016; first published online 9 September 2016

\section{SUMMARY}

The risk ratio quantifies the risk of disease in a study population relative to a reference population. Standard methods of estimation and testing assume a perfect diagnostic test having sensitivity and specificity of $100 \%$. However, this assumption typically does not hold, and this may invalidate naive estimation and testing for the risk ratio. We propose procedures that control for sensitivity and specificity of the diagnostic test, given the risks are measured by proportions, as it is in crosssectional studies or studies with fixed follow-up times. These procedures provide an exact unconditional test and confidence interval for the true risk ratio. The methods also cover the case when sensitivity and specificity differ in the two groups (differential misclassification). The resulting test and confidence interval may be useful in epidemiological studies as well as in clinical and vaccine trials. We illustrate the method with real-life examples which demonstrate that ignoring sensitivity and specificity of the diagnostic test may lead to considerable bias in the estimated risk ratio.

Key words: Exact confidence interval, exact unconditional test, misclassification, prevalence ratio, relative risk.

\section{INTRODUCTION}

The risk ratio or relative risk (RR) quantifies the risk in a study population (say, in those exposed to a risk factor) relative to a reference population. Naive inference assumes that the applied diagnostic procedure is perfect (its sensitivity and specificity is $100 \%$ ), i.e. no misclassification occurs. Unfortunately, this assumption usually does not hold, and ignoring this may result in misleading conclusions. Our aim is to present an exact unconditional test and confidence interval for RR controlling for sensitivity and specificity of the diagnostic test.

\footnotetext{
* Author for correspondence: Dr J. Reiczigel, University of Veterinary Medicine Budapest, István u. 2, 1078 Budapest, Hungary.

(Email: reiczigel.jeno@univet.hu)
}

We address the situation, in which

- risks are quantified by the proportion of those having the condition (disease, recovery, etc.), which is typical in cross-sectional epidemiological studies but may also occur in other study designs;

- two independent random samples are drawn from the two populations;

- there is no classification error in the group definition (i.e. in the exposure);

- sensitivity and specificity of the diagnostic procedure are known (rather than estimated in the same or in another study).

In this case $\mathrm{RR}$ is defined as the ratio of two proportions, $\mathrm{RR}=p_{2} / p_{1}$, where $p_{2}$ is the proportion of diseased in the study group and $p_{1}$ is that in the reference group. Many authors use the term 'prevalence 
ratio' (PR) for this measure preserving the term 'risk ratio' for the incidence ratio [1-3], while others call it 'prevalence risk ratio' (PRR) [4, 5]. In the following text we will use the term risk ratio (RR). In crosssectional studies and in therapeutic or vaccine trials with fixed-length follow-up this is the most natural measure to compare the groups. Guidelines for vaccine studies define vaccine efficacy as $1-R R$, where the reference group is placebo. Despite this, in many studies odds ratios (OR) are calculated just because logistic regression has become a standard analysis tool readily available in most statistical software systems, although using OR instead of RR is repeatedly criticized by statisticians $[1,6-8]$.

While the impact of misclassification on the results of statistical analyses has been studied since the 1950s in the biomedical as well as in the social sciences [9-16], no exact test or confidence interval for the true PR has been proposed. In the next section we describe the proposed procedures, then we present two applications, finally we summarize the properties of the method. $\mathrm{R}$ code for the procedures is available at www2.univet.hu/users/jreiczig/RR_SeSp.

\section{METHODS}

Let us denote for population $i(i=1,2)$ the true prevalence by $p_{i}$, the sensitivity by $S e_{i}$, and the specificity by $S p_{i}$. Then the probability of a positive diagnosis (also called observed or apparent prevalence) in the $i$ th population is $p_{i a}=p_{i} \cdot S e_{i}+\left(1-p_{i}\right) \cdot\left(1-S p_{i}\right)$. This implies that taking independent samples of sizes $n_{1}$ and $n_{2}$ from the two populations, the number of test positives $x_{1}$ and $x_{2}$ follow the binomial distribution with parameters $n_{1}, p_{1 a}$ and $n_{2}, p_{2 a}$. Thus, the relative frequencies $x_{1} / n_{1}$ and $x_{2} / n_{2}$ are estimates of $p_{1 a}$ and $p_{2 a}$, therefore we will denote them by $\hat{p}_{1 a}$ and $\hat{p}_{2 a}$. What we will make use of in the following text is that the parameters $p_{1 a}$ and $p_{2 a}$ are in a one-to-one relationship with the true prevalences $p_{1}$ and $p_{2}$, i.e. a hypothesis about $p_{1}$ and $p_{2}$ can be mapped onto a corresponding hypothesis about $p_{1 a}$ and $p_{2 a}$, and tested using their estimates $\hat{p}_{1 a}$ and $\hat{p}_{2 a}$. The general equation, which describes the relationship between the parameters $p_{i}$ and $p_{i a}$, and allows for $S e_{1} \neq S e_{2}$ and/or $S p_{1} \neq S p_{2}$ is

$$
\begin{aligned}
p_{2 a}= & p_{1 a} \operatorname{RR} \frac{\left(S e_{2}+S p_{2}-1\right)}{\left(S e_{1}+S p_{1}-1\right)} \\
& -\operatorname{RR}\left(1-S p_{1}\right) \frac{\left(S e_{2}+S p_{2}-1\right)}{\left(S e_{1}+S p_{1}-1\right)}+\left(1-S p_{2}\right) .
\end{aligned}
$$

If $S e_{1}=S e_{2}$ and $S p_{1}=S p_{2}$, which can often be assumed in real-life applications, the equation simplifies to

$p_{2 a}=p_{1 a} \mathrm{RR}+(1-S p)(1-\mathrm{RR})$,

where $S p$ denotes the common specificity; and for a perfect diagnostic test, i.e. for $S e_{1}=S e_{2}=S p_{1}=S p_{2}=1$, it reduces to

$p_{2 a}=p_{1 a} \mathrm{RR}$.

Solving the equations (1)-(3) for RR, the following expressions are obtained:

$\mathrm{RR}=\frac{\left(p_{2 a}+S p_{2}-1\right)\left(S e_{1}+S p_{1}-1\right)}{\left(p_{1 a}+S p_{1}-1\right)\left(S e_{2}+S p_{2}-1\right)}$,

$\mathrm{RR}=\left(p_{2 a}+S p-1\right) /\left(p_{1 a}+S p-1\right)$,

$\mathrm{RR}=p_{2 a} / p_{1 a}$.

The point estimates for RR can be obtained by replacing $p_{1 a}$ and $p_{2 a}$ by $\hat{p}_{1 a}$ and $\hat{p}_{2 a}$ in equations (4)-(6). Note that the parameter space for the true prevalences $\left(p_{1}, p_{2}\right)$ is the unit square, whereas that for $\left(p_{1 a}, p_{2 a}\right)$ is a rectangle within the unit square, namely $\left[1-S p_{1}\right.$, $\left.S e_{1}\right] \times\left[1-S p_{2}, S e_{2}\right]$. Note also that the estimates $\left(\hat{p}_{1 a}, \hat{p}_{2 a}\right)$ form a point in the sample space (which is actually a grid of points), rather than in the parameter space. In formula

$\left(\hat{p}_{1 a}, \hat{p}_{2 a}\right) \in\left\{0,1 / n_{1}, 2 / n_{1}, \ldots, 1\right\} \times\left\{0,1 / n_{2}, 2 / n_{2}, \ldots, 1\right\}$.

Assume now that we want to test for $H_{0}: \mathrm{RR}=$ $\mathrm{RR}_{0}$, where $\mathrm{RR}=p_{2} / p_{1}$ is the true risk ratio. This $H_{0}$ is a composite hypothesis, corresponding to a line segment in the parameter space of the true prevalences $p_{1}$ and $p_{2}$, namely the set of points in the unit square satisfying the equation $p_{2}=p_{1} \mathrm{RR}_{0}$. If we map this onto the parameter space of the observed binomials $p_{1 a}$ and $p_{2 a}$, it will form another line segment. Its position depends on $\mathrm{RR}_{0}$ as well as on the sensitivities and specificities according to equations (1)-(3), but it is always located within the rectangle [1 $\left.-S p_{1}, S e_{1}\right] \times\left[1-S p_{2}, S e_{2}\right]$. Figure 1 illustrates the position of this line segment for $H_{0}: \mathrm{RR}=2 \mathrm{de}-$ pending on the sensitivities $S e_{1}, S e_{2}$, and specificities $S p_{1}, S p_{2}$.

Testing for $H_{0}$ is equivalent to testing whether the parameters $p_{1 a}$ and $p_{2 a}$ of the observed independent binomial variables are located on the line segment corresponding to $H_{0}$. As this is also a composite hypothesis, it can be tested applying the intersection-union principle [17], which means that a critical (or 


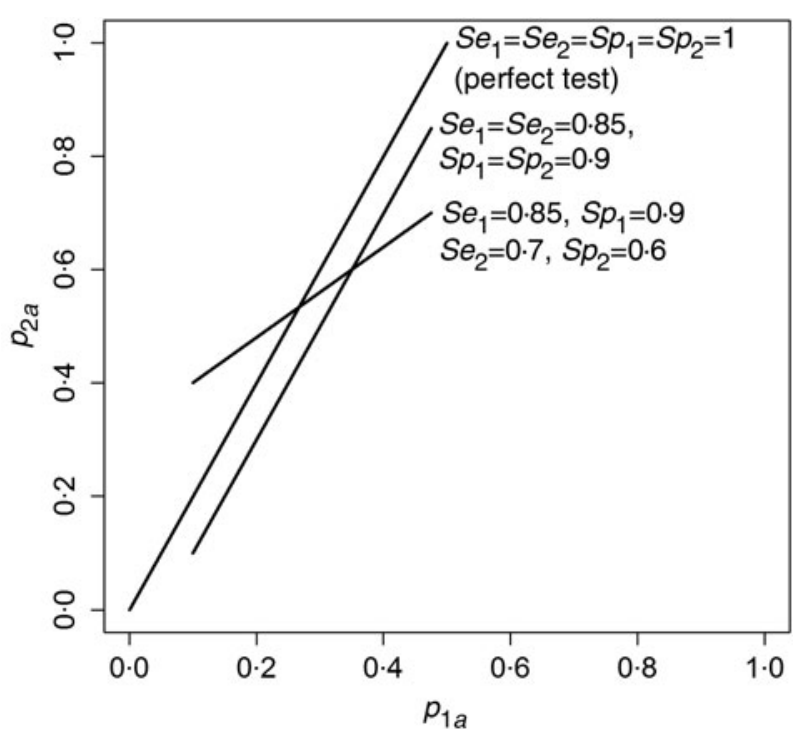

Fig. 1. Lines corresponding to the hypothesis $H_{0}: \mathrm{RR}=2$ in the two-dimensional space of the parameters $p_{1 a}$ and $p_{2 a}$ of the observed binomial variables. The position of the line depends on sensitivity $\left(S e_{1}\right.$ and $\left.S e_{2}\right)$ and specificity $\left(S p_{1}\right.$ and $\left.S p_{2}\right)$ of the test in the two populations.

rejection) region for a composite $H_{0}$ can be obtained by constructing an appropriate critical region for each element of $H_{0}$, and taking the intersection of these regions. The steps of constructing this critical region follow the logic of Reiczigel et al. [18].

(1) For each simple hypothesis $h_{0} \in H_{0}$, i.e. for each point $\left(p_{1 a}, p_{2 a}\right)$ on the line segment representing $H_{0}$, we construct a critical region (rejection region) $C_{h 0}$ in the sample space, consisting of those points, which have probability under $h_{0}$ less than or equal to the probability of the observed point $\left(\hat{p}_{1 a}, \hat{p}_{2 a}\right)$. In formula

$$
\begin{aligned}
C_{h 0}= & \left\{\left(i / n_{1}, j / n_{2}\right): i \in\left\{0, \ldots, n_{1}\right\}, j \in\right. \\
& \left.\left\{0, \ldots, n_{2}\right\}, P_{h 0}\left(i / n_{1}, j / n_{2}\right) \leqslant P_{h 0}\left(\hat{p}_{1 a}, \hat{p}_{2 a}\right)\right\},
\end{aligned}
$$

where $P_{h 0}($ ) denotes the probability of a point (or a set of points) in the sample space, given $h_{0}$ is true. $C_{h 0}$ can be considered as the two-dimensional generalization of that proposed by Sterne [19] for a single binomial proportion. Let $P_{h 0}\left(C_{h 0}\right)$ denote the probability of $C_{h 0}$ under $h_{0}$, and let $M=\max \left\{P_{h 0}\left(C_{h 0}\right), h_{0} \in H_{0}\right\}$.

(2) Next, for each $h_{0} \in H_{0}$ we determine the subset $S_{h 0}$ of the sample space consisting of the points with the smallest probability under $h_{0}$ so that $P_{h 0}\left(S_{h 0}\right) \leqslant M$ but adding any further point to $S_{h 0}$ would result in $P_{h 0}\left(S_{h 0}\right)>M$. Let $C_{H 0}$ denote the intersection of all these subsets, i.e. $C_{H 0}=$ $\cap_{h_{0} \in H_{0}} S_{h 0}$. It is easy to see that $C_{H 0}$ contains $\left(\hat{p}_{1 a}, \hat{p}_{2 a}\right)$ on its boundary.

(3) Finally, the $P$ value is defined as the highest probability of $C_{H 0}$ under $H_{0}$ (i.e. for all simple hypotheses $h_{0}$ in $\left.H_{0}\right)$. In formula, the $P$ value is equal to $\max \left\{P_{h 0}\left(C_{H 0}\right), h_{0} \in H_{0}\right\}$.

Figure 2 illustrates how the resulting critical region for $H_{0}: \mathrm{RR}=2$ depends on sensitivities and specificities, given the two observed prevalences are $\hat{p}_{1 a}=$ $0.575\left(n_{1}=40\right)$ and $\hat{p}_{2 a}=0.667\left(n_{2}=36\right)$.

Confidence intervals for the true RR can be constructed by inverting the above test. That is, lower and upper confidence limits to a given confidence level $(1-\alpha)$ are defined as the smallest and largest true $\mathrm{RR}_{0}$ not rejected by the test, i.e.

$L=\inf \left\{\mathrm{RR}_{0}: p_{\mathrm{RR}_{0}}>\alpha\right\}$ and $U=\sup \left\{\mathrm{RR}_{0}: p_{\mathrm{RR}_{0}}>\alpha\right\}$,

where $p_{\mathrm{RR}_{0}}$ denotes the $P$ value from testing for $H_{0}$ : $\mathrm{RR}=\mathrm{RR}_{0}$. Computationally, $L$ and $U$ are determined by increasing $\mathrm{RR}_{0}$ in small steps and performing the test. Step size may depend on the required precision. In our implementation of the algorithm the default is a multiplicative increment with step size $0 \cdot 001$, i.e. $\mathrm{RR}_{0}$ is increased or decreased as $\mathrm{RR}_{0, \text { next }}=1 \cdot 001 * \mathrm{RR}_{0}$ or $0.999 * R R_{0}$. Figure 3 illustrates the procedure for observed proportions $\hat{p}_{1 a}=0.575\left(n_{1}=40\right), \hat{p}_{2 a}=0.667$ $\left(n_{2}=36\right)$, and $S e_{1}=S e_{2}=0 \cdot 91, S p_{1}=S p_{2}=0 \cdot 8$.

One-tailed testing, i.e. $H_{0}: \mathrm{RR}=\mathrm{RR}_{0}$ against $H_{1}$ : $\mathrm{RR}>\mathrm{RR}_{0}$ (or $H_{1}: \mathrm{RR}<\mathrm{RR}_{0}$ ) is also possible, although there are different options to define this. Perhaps the simplest method is that one side of the line representing $H_{0}$ (i.e. the intersection of the critical region with that half-plane) is removed from the critical region. One-sided confidence intervals (CI) can be derived by inverting this one-sided test.

\section{APPLICATIONS}

\section{Example 1}

Everhart et al. [20] studied the seroprevalence of Helicobacter pylori infection in adults in the United States. The analysis was carried out stratified by age and ethnic group. The infection status was determined by an IgG ELISA assay having 91\% sensitivity and $96 \%$ specificity in all groups. For illustration we now compare the group of the youngest (20-29 years) and the oldest ( $\geqslant 70$ years), in which the observed seroprevalence was $16 \cdot 7 \%$ and $56 \cdot 9 \%$, respectively. For these groups, the ratio of observed prevalences 

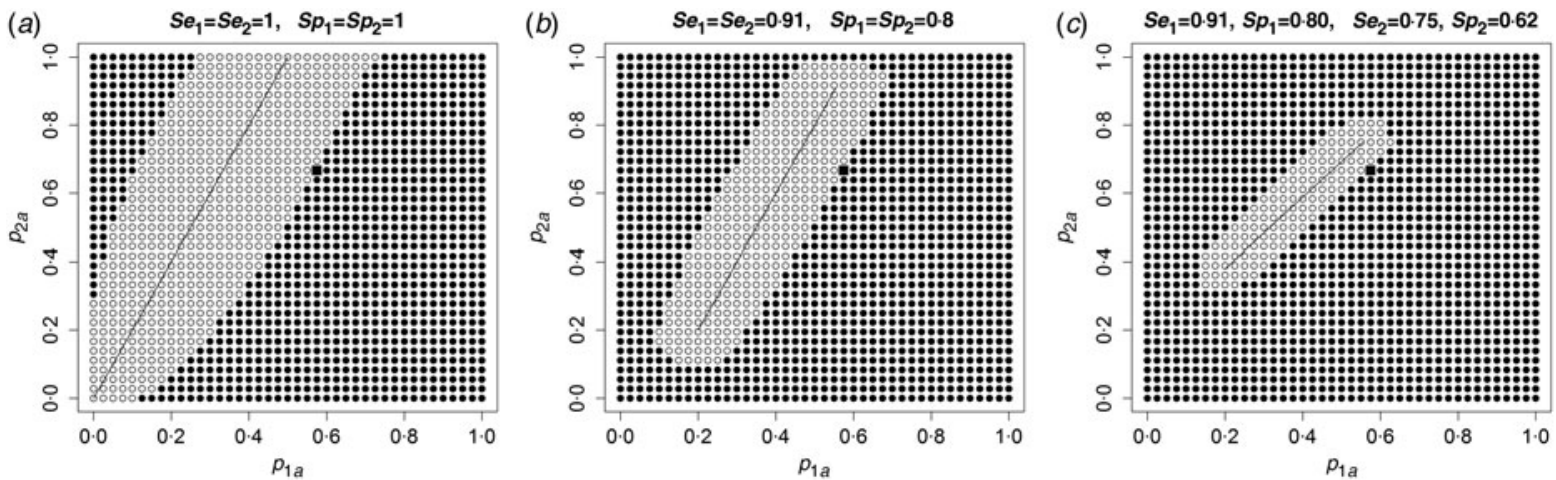

Fig. 2. Critical regions in the sample space for $H_{0}: \mathrm{RR}=2$ with observed proportions $\hat{p}_{1 a}=0 \cdot 575\left(n_{1}=40\right)$ and $\hat{p}_{2 a}=0 \cdot 667$ $\left(n_{2}=36\right)$, depending on the sensitivities and specificities. Black dots form the critical region, the black square represents the observed data. The line shows the location of $H_{0}$ in the parameter space.
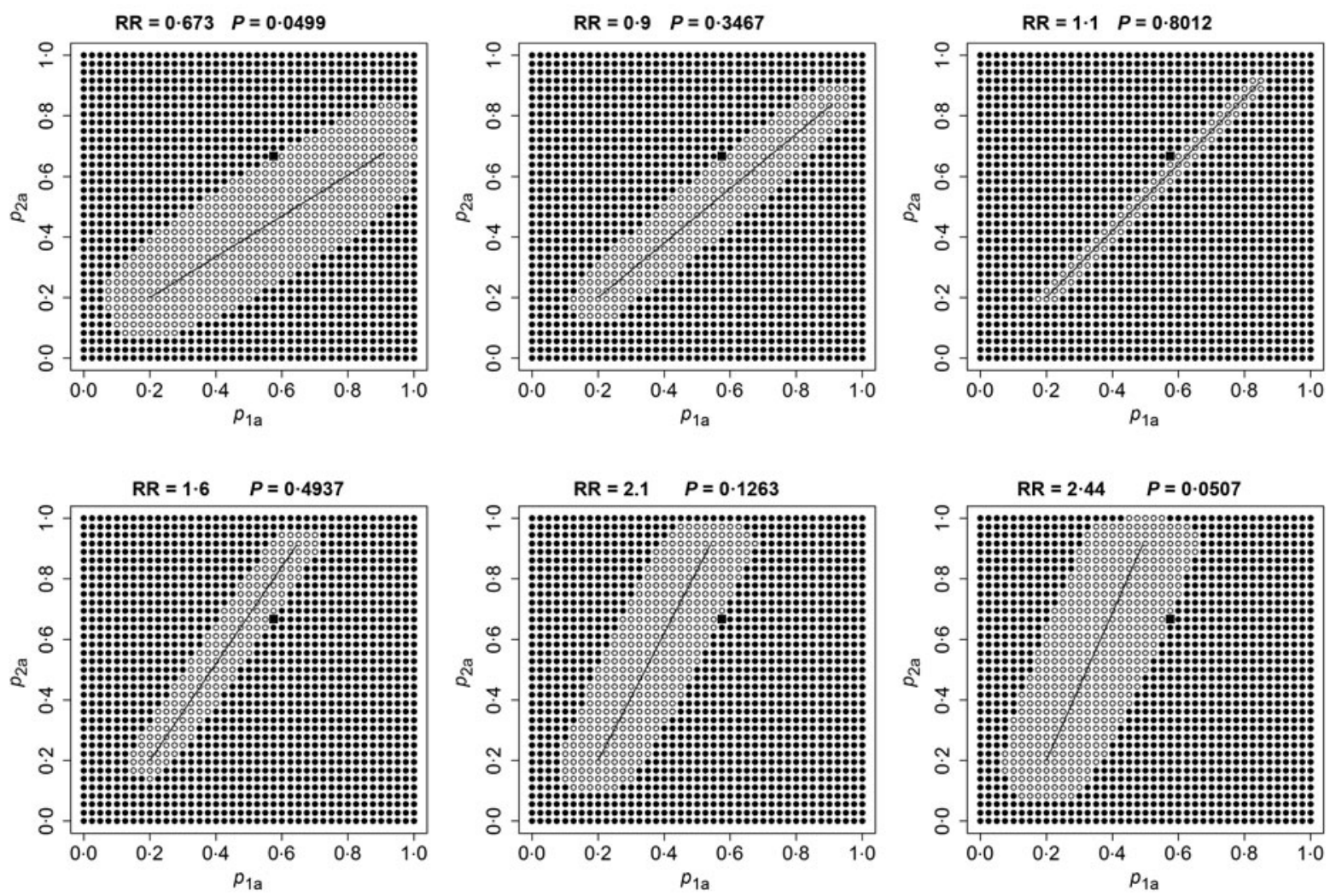

Fig. 3. Illustration of the confidence interval construction for observed proportions $\hat{p}_{1 a}=0 \cdot 575\left(n_{1}=40\right), \hat{p}_{2 a}=0 \cdot 667\left(n_{2}=36\right)$, and $S e_{1}=S e_{2}=0.91, S p_{1}=S p_{2}=0.8$. The confidence limits represent the smallest and largest true risk ratio (RR) not rejected by the test.

is $56 \cdot 9 / 16 \cdot 7=3 \cdot 41(95 \%$ CI $3 \cdot 00-3 \cdot 88)$, whereas the correction for sensitivity and specificity results in the true PR of $4 \cdot 17$ (95\% CI 3·58-4.96).

\section{Example 2}

Suwancharoen et al. [21] conducted a serological survey of leptospirosis in livestock in Thailand using the microscopic agglutination test (MAT) to determine serostatus of the examined animals. Five animal species were included in the study: cattle, buffaloes, pigs, sheep and goats. Infection status of each species was measured by seroprevalence, and all other species were compared to cattle as the reference group by calculating the PRs (which are same as risk ratios). In this study the MAT test was assumed to be perfect, 
Table 1. Prevalence ratios reported in Suwancharoen et al. [21] and adjusted prevalence ratios assuming sensitivity $=0 \cdot 76$ and specificity $=0.97$ (same in all groups), as reported by Cumberland et al. [22]

\begin{tabular}{llllll}
\hline \hline Species & $N$ & Observed prevalence & Unadjusted PR (95\% CI) & True prevalence & Adjusted PR (95\% CI) \\
\hline Cattle & 9288 & $9 \cdot 9 \%$ & (reference group) & $9 \cdot 4 \%$ & (reference group) \\
Buffaloes & 1376 & $30 \cdot 5 \%$ & $3 \cdot 08(2 \cdot 79-3 \cdot 41)$ & $37 \cdot 6 \%$ & $3 \cdot 99(3 \cdot 45-4 \cdot 60)$ \\
Pigs & 1898 & $10 \cdot 8 \%$ & $1 \cdot 09(0 \cdot 95-1 \cdot 26)$ & $10 \cdot 7 \%$ & $1 \cdot 13(0 \cdot 90-1 \cdot 42)$ \\
Sheep & 1110 & $4 \cdot 7 \%$ & $0 \cdot 47(0 \cdot 36-0 \cdot 62)$ & $2 \cdot 3 \%$ & $0 \cdot 24(0 \cdot 06-0 \cdot 49)$ \\
Goats & 516 & $7 \cdot 9 \%$ & $0 \cdot 80(0 \cdot 60-1 \cdot 09)$ & $6 \cdot 8 \%$ & $0 \cdot 72(0 \cdot 37-1 \cdot 17)$ \\
\hline
\end{tabular}

PR, Prevalence ratio; CI, confidence interval.

as this test is usually regarded to be the gold standard test. However, other studies found that the sensitivity of the MAT test is far below $100 \%$ [22-25]. Cumberland et al. [22] found the sensitivity to be $30 \%$ for first acute-phase specimens, $63 \%$ for second acute-phase specimens, and $76 \%$ for convalescent specimens. At the same time specificity was $99 \%, 98 \%$, and 97\%, respectively. Limmathurotsakul et al. [25] estimated the sensitivity of MAT by a Bayesian analysis and found it to be $49 \cdot 8 \%$. These findings indicate the need for an adjustment of the PR estimates.

If we consider the most optimistic scenario of these, i.e. $S e=76 \%$ and $S p=97 \%$ [22], we obtain the adjusted PRs shown in Table 1. The difference between the unadjusted and adjusted PR is far from negligible, for example in case of sheep the adjusted ratio is less than half of the unadjusted one. In case of buffaloes, adjusted and unadjusted $95 \%$ CIs do not even overlap.

\section{DISCUSSION}

If sensitivity and/or specificity of the diagnostic test is $<100 \%$, the observed and true prevalence may differ from each other, influencing also estimation and testing of relative risk measured by the PR. We proposed an exact unconditional test and CIs for the true PR. The method can be applied even if the sensitivities and specificities differ in the two groups, for example if patients are diagnosed by different methods or some sort of differential misclassification occurs.

Taking sensitivity and specificity into account may either increase or decrease the $P$ value compared to the one obtained without considering sensitivity and specificity. For instance, consider testing for $H_{0}$ : $\mathrm{RR}=2$ with observed prevalences $\hat{p}_{1 a}=0.48\left(n_{1}=50\right)$ and $\hat{p}_{2 a}=0.62\left(n_{2}=50\right)$. Assuming $S e_{1}=S e_{2}=S p_{1}=$ $S p_{2}=1$ results in $P=0.0251$, for $S e_{1}=S e_{2}=0.8$ and $S p_{1}=S p_{2}=1$ the $P$ value increases to $P=0.0474$, whereas for $S e_{1}=S e_{2}=0.6$ and $S p_{1}=S p_{2}=1$ a smaller $P$ value of 0.0155 is obtained.

It may occur that the observed data contradict the given sensitivity and specificity. Let us assume, for example, that a certain diagnostic test is known to have $S e=0.8$ and $S p=1$ and using this test we observe 90 positives out of 100 . This observation is very unlikely even if the true prevalence is $100 \%$, since under these conditions the observed variable has a binomial distribution with $n=100, P=0 \cdot 8$, and the probability that it is $\geqslant 90$ is as low as $0 \cdot 0057$. The same problem may arise if the number of positives is much less than the expected minimum assuming the given sensitivity and specificity. In such cases one should consider the possibility that sensitivity and/or specificity data are incorrect.

The proposed method can be further developed in several directions. First, one can take into account misclassification also in the group definition, i.e. in the exposure. Brenner et al. [26] investigated this for the incidence ratio in a cohort study. Going further, similar methods could be worked out for models with several predictors, of which the categorical ones may also be affected by misclassification. Some results are known on correcting the OR obtained from logistic regression [27, 28] but similar results still lack for the PR.

There has been a long debate whether the PR or the $\mathrm{OR}$ is more appropriate to quantify the risk in the study group relative to the control group in a study design in which both of them can be calculated [1, 7, 29, 30]. Savu et al. [8] stated that RR or PR is more intuitively interpretable than the OR. In spite of this, many studies report OR estimates, even if the design permits calculation of RR or PR. Petersen \& Deddens [31] emphasized that in cross-sectional studies, in particular when the disease is not rare, it is preferable to use PR instead of OR. Anyway, it is worth noting that our method can quite easily be adapted 
to testing the $\mathrm{OR}$ or the risk difference (RD), as the hypotheses $H_{0}: \mathrm{OR}=\mathrm{OR}_{0}$ and $H_{0}: \mathrm{RD}=\mathrm{RD}_{0}$ also correspond to a subset of the parameter space $[0,1] \times[0,1]$.

Another direction of potential improvement of the proposed methods is to extend them to the case when sensitivity and specificity are not taken as known but estimated from other samples, which may increase the variance of the RR estimate. This is analogous to the problem solved by Lang \& Reiczigel [32] for estimating prevalence.

\section{ACKNOWLEDGEMENTS}

This work was supported by the Hungarian National Research Fund (grant no. OTKA K108571) and by the Research Faculty Grant 2015 of the Szent István University, Faculty of Veterinary Science.

\section{DECLARATION OF INTEREST}

None.

\section{REFERENCES}

1. Lee J, Chia KS. Estimation of prevalence rate ratios for cross sectional data: an example in occupational epidemiology. British Journal of Industrial Medicine 1993; 50: $861-862$.

2. Thompson ML, Myers JE, Kriebel D. Prevalence odds ratio or prevalence ratio in the analysis of cross sectional data: what is to be done? Occupational and Environmental Medicine 1998; 55: 272-277.

3. Santos CA, et al. Estimating adjusted prevalence ratio in clustered cross-sectional epidemiological data. $B M C$ Medical Research Methodology 2008; 8: 80.

4. Lui K-J. Statistical Estimation of Epidemiological Risk. John Wiley \& Sons, Hoboken, NJ, USA, 2004, 214 pp.

5. Holmes Jr. L, Opara F. Concise Biostatistical Principles \& Concepts: Guidelines for Clinical and Biomedical Researchers. AuthorHouse, Bloomington, USA, 2014, $391 \mathrm{pp}$.

6. Axelson O, Fredriksson M, Ekberg K. Use of the prevalence ratio $\mathrm{v}$ the prevalence odds ratio as a measure of risk in cross sectional studies. Occupational and Environmental Medicine 1994; 51: 574.

7. Nurminen M. To use or not to use the odds ratio in epidemiologic analyses? European Journal of Epidemiology 1995; 11: 365-371.

8. Savu A, Liu Q, Yasui Y. Estimation of relative risk and prevalence ratio. Statistics in Medicine 2010; 29: 22692281.

9. Bross I. Misclassification in $2 \times 2$ tables. Biometrics 1954; 10: 478-486.

10. Diamond EL, Lilienfeld AM. Effects of errors in classification and diagnosis in various types of epidemiological- studies. American Journal of Public Health and the Nation's Health 1962; 52: 1137-1144.

11. Mote VL, Anderson RL. An investigation of the effect of misclassification on the properties of chi-2-tests in the analysis of categorical data. Biometrika 1965; 52: 95-109.

12. Assakul K, Proctor CH. Testing independence in twoway contingency tables with data subject to misclassification. Psychometrika 1967; 32: 67-76.

13. Copeland KT, et al. Bias due to misclassification in the estimation of relative risk. American Journal of Epidemiology 1977; 105: 488-495.

14. Reiczigel J, Földi J, Ózsvári L. Exact confidence limits for prevalence of a disease with an imperfect diagnostic test. Epidemiology and Infection 2010; 138: 1674-1678.

15. Porter KA, et al. Uncertain outcomes: adjusting for misclassification in antimalarial efficacy studies. Epidemiology and Infection 2011; 139: 544-551.

16. Jackson ML, Rothman KJ. Effects of imperfect test sensitivity and specificity on observational studies of influenza vaccine effectiveness. Vaccine 2015; 33: 1313-1316.

17. Casella G, Berger RL. Statistical Inference. Duxbury Press, Belmont, CA, USA, 1990. 660 pp.

18. Reiczigel J, Abonyi-Tóth Z, Singer J. An exact confidence set for two binomial proportions and exact unconditional confidence intervals for the difference and ratio of proportions. Computational Statistics and Data Analysis 2008; 52: 5046-5053.

19. Sterne TE. Some remarks on confidence or fiducial limits. Biometrika 1954; 41: 275-278.

20. Everhart JE, et al. Seroprevalence and ethnic differences in Helicobacter pylori infection among adults in the United States, Journal of Infectious Diseases 2000; 181: 1359-1363.

21. Suwancharoen D, et al. Serological survey of leptospirosis in livestock in Thailand. Epidemiology and Infection 2013; 141: 2269-2277.

22. Cumberland P, Everard CO, Levett PN. Assessment of the efficacy of an IgM-ELISA and microscopic agglutination test (MAT) in the diagnosis of acute leptospirosis. American Journal of Tropical Medicine and Hygiene 1999; 61: 731-734.

23. Vijayachari P, Sugunan AP, Sehgal SC. Evaluation of microscopic agglutination test as a diagnostic tool during acute stage of leptospirosis in high and low endemic areas. Indian Journal of Medical Research 2001; 114: 99.

24. Smythe LD, et al. The microscopic agglutination test (MAT) is an unreliable predictor of infecting Leptospira serovar in Thailand. American Journal of Tropical Medicine and Hygiene 2009; 81: 695-697.

25. Limmathurotsakul D, et al. Fool's gold: why imperfect reference tests are undermining the evaluation of novel diagnostics: a reevaluation of 5 diagnostic tests for leptospirosis. Clinical Infectious Diseases 2012; 55: 322-331.

26. Brenner H, Savitz DA, Gefeller O. The effects of joint misclassification of exposure and disease on epidemiologic measures of association. Journal of Clinical Epidemiology 1993; 46: 1195-1202.

27. Magder LS, Hughes JP. Logistic regression when the outcome is measured with uncertainty. American Journal of Epidemiology 1997; 146: 195-203. 
28. Cheng KF, Hsueh HM. Correcting bias due to misclassification in the estimation of logistic regression models. Statistics and Probability Letters 1999; 44: 229-240.

29. Pearce N. Effect measures in prevalence studies. Environmental Health Perspectives 2004; 112: 1047-1050.

30. Reichenheim ME, Coutinho ESF. Measures and models for causal inference in cross-sectional studies: arguments for the appropriateness of the prevalence odds ratio and related logistic regression. BMC Medical Research Methodology 2010; 10: 66.

31. Petersen MR, Deddens JA. A comparison of two methods for estimating prevalence ratios. BMC Medical Research Methodology 2008; 8: 9

32. Lang Z, Reiczigel J. Confidence limits for prevalence of disease adjusted for estimated sensitivity and specificity. Preventive Veterinary Medicine 2014; 113: 13-22. 